\title{
Safety and efficacy of sintilimab combined with oxaliplatin/capecitabine as first-line treatment in patients with locally advanced or metastatic gastric/gastroesophageal junction adenocarcinoma in a phase $\mathrm{lb}$ clinical trial
}

Haiping Jiang ${ }^{1}$, Yulong Zheng ${ }^{1}$, Jiong Qian ${ }^{1}$, Chenyu Mao ${ }^{1}$, Xin Xu ${ }^{1}$, Ning $L_{i}{ }^{1}$, Cheng Xiao ${ }^{1}$, Huan Wang ${ }^{1}$, Lisong Teng ${ }^{2}$, Hui Zhou ${ }^{3}$, Shuyan Wang ${ }^{3}$, Donglei Zhu ${ }^{3}$, Bo Peng ${ }^{4}$, Lin Shen ${ }^{5}$ and Nong Xu ${ }^{1 *}$

\begin{abstract}
Background: Sintilimab blocks the interaction between programmed death-1 (PD-1) and its ligands. The safety and efficacy of sintilimab combined with oxaliplatin/capecitabine (CapeOx) as first-line treatment were evaluated in patients with gastric (G)/gastroesophageal junction (GEJ) adenocarcinoma in a phase Ib clinical trial.

Methods: Patients with locally advanced or metastatic G/GEJ adenocarcinoma without previous systemic treatment were enrolled as one cohort of a multi-cohort study. Sintilimab was administered at a dose of $200 \mathrm{mg}$ intravenously (IV) in combination with CapeOx (1000 mg/m² capecitabine orally, bid, D1-14 and $130 \mathrm{mg} / \mathrm{m}^{2}$ oxaliplatin IV, D1) every 21 days for up to 6 cycles. After combination treatment, patients continued to receive sintilimab (200 mg) at 3 weekly intervals as maintenance therapy until progressive disease (PD), unacceptable toxicity, withdrawal of informed consent, or for up to 24 months. Adverse events (AEs) were monitored to assess safety in terms of their frequency, intensity and causality. The efficacy endpoints included the objective response rate (ORR), disease control rate (DCR), progression-free survival (PFS) and overall survival (OS). Tumor mutation burden (TMB) was evaluated for its association with clinical response.
\end{abstract}

Results: A total of 20 patients were enrolled and received sintilimab plus CapeOx. All patients reported treatment-related AEs (TRAEs). Grade 3-4 TRAEs were found in 11 (55.0\%) patients. Seventeen patients obtained partial response and the ORR was 85.0\% (95\% Cl: 62.1-96.8\%). Three (15.0\%) had stable disease and DCR was 100.0\% (95\% Cl: 83.2-100.0\%). As data cutoff of May 1, 2019, the median follow-up was 7.8 months. The median PFS was 7.5 months (95\% Cl: 6.2-9.4) and median OS had not been reached. The OS rates at 6 months and 12 months were 100.0 and $68.0 \%$. No association was observed between TMB and efficacy.

(Continued on next page)

\footnotetext{
* Correspondence: Nongxu.zhjph@outlook.com

'Department of Medical Oncology, The First Affiliated Hospital, School of

Medicine, Zhejiang University, No. 79, 86 Qingchun Road, Hangzhou 310003,

China

Full list of author information is available at the end of the article
}

(c) The Author(s). 2020 Open Access This article is licensed under a Creative Commons Attribution 4.0 International License, which permits use, sharing, adaptation, distribution and reproduction in any medium or format, as long as you give appropriate credit to the original author(s) and the source, provide a link to the Creative Commons licence, and indicate if changes were made. The images or other third party material in this article are included in the article's Creative Commons licence, unless indicated otherwise in a credit line to the material. If material is not included in the article's Creative Commons licence and your intended use is not permitted by statutory regulation or exceeds the permitted use, you will need to obtain permission directly from the copyright holder. To view a copy of this licence, visit http://creativecommons.org/licenses/by/4.0/. The Creative Commons Public Domain Dedication waiver (http://creativecommons.org/publicdomain/zero/1.0/) applies to the data made available in this article, unless otherwise stated in a credit line to the data. 
(Continued from previous page)

Conclusions: Sintilimab combined with CapeOx as first-line treatment demonstrated acceptable safety and promising efficacy.

Trial registration: ClinicalTrials.gov, NCT02937116. Registered 8 October 2016.

Keywords: Sintilimab, Capecitabine, Oxaliplatin, Gastric/gastroesophageal junction adenocarcinoma, Tumor mutation burden

\section{Background}

The fifth most commonly diagnosed cancer worldwide is gastric cancer (GC), accounting for about 33\% of cancer-related deaths globally and the third most common cancer in China with almost half of worldwide new GC cases occurring in China annually $[1,2]$. The standard treatments exhibit regional differences among western countries, Japan/Korea and China, which are considered to be associated with different screening and early detection methods as well as different biological behaviors, disease characteristics and ethnicity [3-5].

Surgical resection is the only radical therapy for gastric/gastroesophageal junction (G/GEJ) cancer. However, systemic chemotherapy is an alternative main therapy for G/GEJ cancer because of the high relapse rate after post-resection surgery and for the many patients diagnosed at an advanced-stage. For advanced G/GEJ cancer, first-line treatment mainly involves platinum-based chemotherapy using a combination of two or three drugs (trastuzumab is given to patients whose tumor is human epidermal growth factor receptor-2 (HER2) positive), but the overall survival (OS) is disappointing, since the maximum OS time has been reported to be 13.8 months [6-10]. Any potential novel drug that will increase patient survival times is urgently needed, in particular for first-line treatment.

Immune checkpoint inhibitor treatment is a new approach for tumor immunotherapy [11, 12]. The treatment diminishes the immune system tolerance to tumor cells and improves the effective identification and eradication of tumor cells by blocking $\mathrm{T}$ cell inhibition [13]. The programmed death-1 (PD-1) antibody specifically binds to PD-1, thereby inhibiting apoptosis of antigen-specific $\mathrm{T}$ cells and thus reducing regulatory $\mathrm{T}$ cell (Treg) apoptosis by inhibiting the activation of PD-L1 $[14,15]$.

The efficacy of anti-PD-1 antibodies monotherapy in patients who had prior chemotherapy for advanced GC has been demonstrated and supported by several trials. In the KEYNOTE-012 and KEYNOTE-059 trials, pembrolizumab monotherapy showed objective response rates (ORR) of $22 \%(n=36)$ [16] and 15.5\% $(n=148)$ [17], respectively in PD-L1 positive advanced GC patients after at least two prior systemic therapies. Based on such results, the Food and Drug Administration approved pembrolizumab for third-line treatment of patients with recurrent or advanced GC. In the ATTR ACTION-2 study, nivolumab monotherapy improved OS from 4.1 to 5.3 months (hazard ratio $0.63,95 \%$ CI: $0.51-0.78 ; P<0.000)$, compared with a placebo in advanced GC that was refractory or intolerant to previous treatment regimens [18].

However, between 30 and $60 \%$ of patients exhibit no response to PD-1 blockade, which is considered to be associated with $\mathrm{T}$ cell exclusion or exhaustion or inadequate $\mathrm{T}$ cell trafficking and many immunosuppressive factors accumulate in the tumor microenvironment [19]. New therapy regimens that improve the response and long-term efficacy are desperately needed. The efficacy of anti-PD-1 therapy in combination with chemotherapy has been confirmed in non-small-cell lung cancer [20, 21]. In addition to direct tumor killing, conventional cytotoxic chemotherapy has demonstrated immunoregulatory properties by enhancing tumor antigenicity, disturbing immune suppressive pathways, inducing immunogenic cell death, and increasing effector T-cell reactions [22]. It is safe to hypothesize that anti-PD-1 antibodies in combination with chemotherapy may further improve the clinical outcomes of patients with advanced GC. Sintilimab is a highly selective, monoclonal IgG4 antibody that inhibits interactions between PD-1 and its ligands, with strong anti-tumor response [23]. A phase 1a study for dose escalation, has demonstrated the tolerance and pharmacological activity of sintilimab in patients with advanced-stage solid tumors, but there is limited evidence for the efficacy of antibodies against PD-1 plus chemotherapy in Chinese G/GEJ adenocarcinoma patients. Thus, the present trial was conducted to investigate the safety and efficacy of sintilimab combined with CapeOx as first-line therapy for a cohort of patients with G/GEJ adenocarcinoma.

\section{Methods}

\section{Study design and patients}

The present study was an open label, multicenter, phase Ib study to evaluate the safety and efficacy of sintilimab in 6 cohorts of patients with solid tumors. Patients (age range 18-70 years) with cytologically or histologically confirmed unresectable G/GEJ adenocarcinoma were enrolled in the G/GEJ cohort. Tumor, nodes and metastases (TNM) staging has been evaluated according to the Union for International Cancer Control (UICC) TNM classification 8th 
edition [24]. The patients had received no previous systemic treatment for advanced disease or had disease progression (PD) more than 6 months after systemic adjuvant therapy. Other major inclusion criteria were: at least one measurable lesion as defined by the Response Evaluation Criteria in Solid Tumor (RECIST version 1.1) criteria; score 0 or 1 for Eastern Tumor Collaborative Group Performance Status (ECOG-PS); adequate organ and bone marrow functions and life expectancy $\geq 12$ weeks. Patients with amplification or overexpression of the HER2 gene were excluded from the trial. Appendix 1 contains a complete list of all inclusion and exclusion criteria.

The institutional review boards of all centers approved the protocols and the study was carried out in strict accordance with the declaration of Helsinki principles; all participating patients signed consent forms before taking part.

\section{Procedures}

According to NCCN guideline, the preferred first-line chemotherapy regimens for advanced gastric cancer are fluorouracil or capecitabine combined with cisplation or oxaliplatin [25]. However, the results from the REAL-2 study [26] revealed significant clinical benefit of the oxaliplatin/capecitabine (CapeOx) regimen which led to the longest OS time of 11.2 months, compared with other regimens. Oxaliplatin produces less renal toxicity, there is no requirement for hydration and it has a lower emetic potential compared to cisplatin, while capecitabine has no requirement for continuous intravenous (IV) infusion and is administered orally, which should ensure an improved quality of life for patients in their homes. Therefore, a CapeOx regimen has been chosen. During the combination phase, enrolled patients were given sintilimab in combination with CapeOx for up to 6 cycles (every 3 weeks). Each cycle consisted of intravenous sintilimab $(200 \mathrm{mg})$ plus oxaliplatin $\left(130 \mathrm{mg} / \mathrm{m}^{2}\right)$ on day 1 and capecitabine (1000 $\mathrm{mg} / \mathrm{m}^{2}$ twice daily orally) from day 1 to day 14 . After combination treatment, patients without PD continued to receive sintilimab $(200 \mathrm{mg})$ at 3 weekly intervals as maintenance therapy until PD, unacceptable toxic effects, withdrawal of informed consent, or for up to 24 months.

\section{Study assessments}

Adverse events (AEs) were monitored for 90 days after the last administration of a treatment dose. Responses were assessed by computed tomography (CT) or magnetic resonance imaging (MRI), every 9 weeks until PD, new treatment initiation, withdrawal of informed consent, or death.

\section{Endpoints}

Safety was assessed as collected AEs according to their type, frequency, causality and severity grading defined by the National Cancer Institute Common Terminology Criteria (CTCAE) ver. 4.03. The efficacy endpoints were the ORR, disease control rate (DCR), time to response (TTR), duration of response (DOR), progression free survival (PFS) and OS. Efficacy was determined by an investigator according to RECIST v1.1 guidelines.

Exploratory endpoints were to evaluate the correlation of tumor mutation burden (TMB) with clinical efficacy.

\section{Tumor mutation burden analysis}

The tumor biopsies and blood samples were collected at baseline. DNA sequences were extracted from biopsies of tumors with matched blood samples and submitted for next generation sequencing using a designed 1622gene panel (Genecast, Beijing, China). TMB was determined by analysis of the quantity of somatic mutations per megabase $(\mathrm{Mb})$. Median TMB was used as a cut-off to define a tumor as high-TMB (H-TMB) and low-TMB (L-TMB).

\section{Statistical analysis}

All patients who received at least one study treatment were included in the safety and efficacy analyses. AEs were coded following the Medical Dictionary for Regulatory Activity and tabulated by system organ class and preferred terms. Causality between AEs and the study treatment was assessed by the investigator. ORR was calculated as the proportion of patients who had achieved a complete response (CR) or partial response (PR) and the 95\% CIs were evaluated by the binomial distribution. DCR was calculated as the proportion of patients who obtained PR, CR and stable disease (SD) and data are presented with the 95\% CIs. Median DOR, TTR, PFS, OS and the PFS and OS rates at 6 and 12 months were determined using the Kaplan-Meier methodology. Fisher's test was used to compare the ORRs between patients with $\mathrm{H}-\mathrm{TMB}$ and L-TMB.

\section{Results}

From 26 Dec, 2017 to 17 Oct 2018, 25 patients were screened and 20 were enrolled in the G/GEJ adenocarcinoma cohort (Fig. 1). The median interval between initial diagnosis and screening was 14 days (range 7-611). Most patients (80.0\%) had metastatic disease status and 11 (55.0\%) had ECOG scores of 1 (Table 1). The TNM stage summary is shown in Table 1 and the staging of each patient in Supplementary Table 1.

At data cutoff on May 1, 2019, the median follow-up time was 7.8 months (range 6.2-12.3). The median treatment duration was 6.2 months (range 2.1-10.4). All patients received more than 4 cycles of treatment, with 


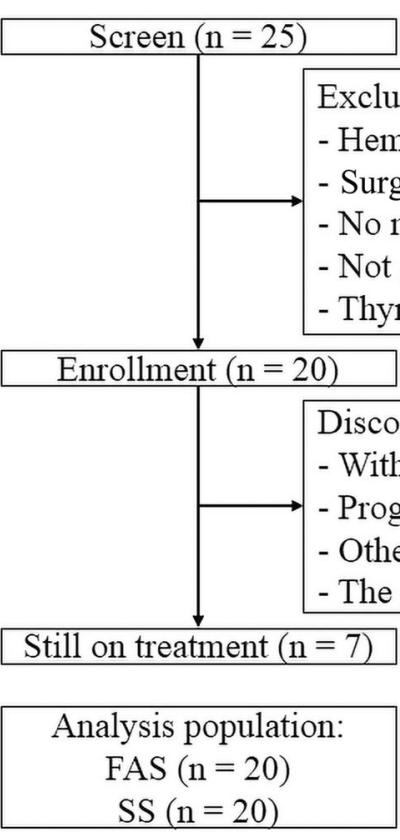

Fig. 1 Flowchart of the study

the median doses of received sintilimab being 9.5 (range $4-16)$.

\section{Safety}

All of the 20 patients reported at least one treatmentrelated adverse event (TRAE), and the most common TRAE was platelet count decreased $(n=16,80.0 \%)$. Grade 3 or 4 treatment-related AEs (TRAEs) occurred in $11(55.0 \%)$ patients, the most common also being a platelet count decreased $(n=9,45.0 \%)$ (Table 2). No TRAE was fatal and 1 patient discontinued the treatment due to treatment-related Grade 3 hepatic function abnormal. Sintilimab-related AEs occurred in 17 (85.0\%) patients. Grade 3-4 sintilimab-related AEs occurred in 5 (25.0\%) patients, the most common being platelet count decreased $(n=3,15.0 \%)$ (Supplementary Table 2). Chemotherapy-related AEs were found in all patients. Grade 3-4 chemotherapy-induced AEs were found in 11 patients $(55.0 \%)$, the most common being platelet count decreased $(n=9,45.0 \%)$ (Supplementary Table 3). Five patients reported treatment-related serious adverse events: platelet count decreased $(n=4)$, abnormal hepatic function $(n=1)$, hypothyroidism $(\mathrm{n}=1)$, pneumonitis $(\mathrm{n}=1)$ and autoimmune colitis $(\mathrm{n}=1)$.

\section{Efficacy}

All 20 patients experienced a decrease in the sum of their target lesions (Fig. 2a) and in the majority the lesions kept smaller than at baseline (Fig. 2b). The median TTR was 2.1 months (95\% CI: 2.0-2.1) and the median DOR was 5.9 months (95\% CI: 4.8-7.2). According to the best tumor response following RECIST 1.1 guidelines, 17 patients reached a PR $(85.0 \%$ (95\% CI: $62.1-$ $96.8 \%)$ ) and $15(75.0 \%)$ patients obtained a confirmed objective response i.e. by two continuous PRs at intervals of 4 weeks. In addition, 3 patients had SD and DCR was 100.0\% (95\% CI: 83.2-100.0\%) (Table 3).

One patient achieved a $\mathrm{CR}$ after the primary analysis by May 1,2019 . This patient began the study treatment on October 12, 2018 and completed 15 cycles of treatment before CR.

The median PFS time was 7.5 months (95\% CI: $6.2-$ 9.4) and the 6 month PFS rate was $88.0 \%$. Median OS was not reached and the 6-month and 12-month OS rates were 100.0 and $68.0 \%$, respectively (Fig. 2c, d).

\section{Tumor mutation burden}

Valid results were obtained from 20 patients. The median TMB value was $1.77 \mathrm{Mb}$. The ORR was $100.0 \%$ (95\% CI: 69.2-100.0\%) in 10 patients with H-TMB, and $70.0 \%$ (95\% CI: 34.8-93.3\%) in patients with LTMB. No significant difference in clinical responses were found between $\mathrm{H}-\mathrm{TMB}$ and L-TMB patients $(P=0.211)$ (Fig. 2e).

\section{Discussion}

In the present study, the results from the G/GEJ adenocarcinoma cohort in a Phase Ib study demonstrated manageable safety and favorable anti-tumor activity of sintilimab combined with a CapeOx regimen as first-line treatment for unresectable advanced metastatic G/GEJ adenocarcinoma. 
Table 1 Demographics and disease characteristics

\begin{tabular}{|c|c|}
\hline & All patients $(N=20)$ \\
\hline Age (median, range) in years & 59.8 (36.9 to 69.3) \\
\hline \multicolumn{2}{|l|}{ Gender (n, \%) } \\
\hline Male & $18(90.0)$ \\
\hline Female & $2(10.0)$ \\
\hline \multicolumn{2}{|l|}{ ECOG PS (n, \%) } \\
\hline 0 & $9(45.0)$ \\
\hline 1 & $11(55.0)$ \\
\hline $\begin{array}{l}\text { Time since initial diagnosis } \\
\text { (median, range) in days }\end{array}$ & $14(7-611)$ \\
\hline \multicolumn{2}{|l|}{ Disease status (n, \%) } \\
\hline Locally advanced & $4(20.0)$ \\
\hline Metastatic & $16(80.0)$ \\
\hline \multicolumn{2}{|l|}{ Location of the primary tumor $(n, \%)$} \\
\hline Upper & $7(35.0)$ \\
\hline Middle & $6(30.0)$ \\
\hline Lower & $7(35.0)$ \\
\hline \multicolumn{2}{|l|}{ TNM staging $(n, \%)$} \\
\hline T3 & $3(15.0)$ \\
\hline T4 & $11(55.0)$ \\
\hline Tx & $6(30.0)$ \\
\hline MO & $4(20.0)$ \\
\hline M1 & $16(80.0)$ \\
\hline N1 & $1(5.0)$ \\
\hline N2 & $3(15.0)$ \\
\hline N3 & $6(30.0)$ \\
\hline Nx & $10(50.0)$ \\
\hline \multicolumn{2}{|l|}{ Histology (n, \%) } \\
\hline Poorly differentiated adenocarcinoma & $11(55.0)$ \\
\hline Moderately differentiated adenocarcinoma & $5(25.0)$ \\
\hline Unknown differentiated adenocarcinoma & $4(20.0)$ \\
\hline
\end{tabular}

ECOG Eastern Cooperative Oncology Group, $T$ tumor, $N$ node, $M$ metastasis

In terms of safety, the incidence and severity of TRAEs with sintilimab and CapeOx were generally consistent with those of known toxic effects of conventional chemotherapy [26-28] and previously reported side effects of other anti-PD-1 antibody combined with chemotherapy regimens [29, 30]. Platelet count, white blood cell count and neutrophil count decreases were most commonly and mostly grade 1 to 2 reported TRAEs and are expected AEs associated with CapeOx [26-28]. Only 1 patient reported discontinuation of investigational drug application due to a TRAE (abnormal hepatic function). No treatmentrelated death occurred in this study and in general, the addition of sintilimab to CapeOx showed a manageable safety profile and did not bear extra safety risks.
Table 2 Treatment-related adverse events (TRAEs)

\begin{tabular}{lll}
\hline & All grade & Grade 3-4 \\
\hline All TRAEs (n) & $\mathbf{2 0 ( 1 0 0 . 0 )}$ & $\mathbf{1 1}(\mathbf{5 5 . 0})$ \\
Platelet count decreased & $16(80.0)$ & $9(45.0)$ \\
White blood cell count decreased & $10(50.0)$ & $0(0.0)$ \\
Neutrophil count decreased & $10(50.0)$ & $2(10.0)$ \\
Hypothyroidism & $6(30.0)$ & $0(0.0)$ \\
Rash & $5(25.0)$ & $0(0.0)$ \\
Alanine aminotransferase increased & $5(25.0)$ & $0(0.0)$ \\
Aspartate aminotransferase increased & $4(20.0)$ & $0(0.0)$ \\
Anemia & $4(20.0)$ & $0(0.0)$ \\
Hepatic function abnormal & $3(15.0)$ & $1(5.0)$ \\
Vomiting & $3(15.0)$ & $0(0.0)$ \\
Nausea & $2(10.0)$ & $0(0.0)$ \\
Hyperchlorhydria & $2(10.0)$ & $0(0.0)$ \\
Thyroid function test abnormal & $2(10.0)$ & $0(0.0)$ \\
Hypokalemia & $2(10.0)$ & $1(5.0)$ \\
Hypesthesia & $2(10.0)$ & $0(0.0)$ \\
Pyrexia & $2(10.0)$ & $0(0.0)$ \\
Proteinuria & $2(10.0)$ & $0(0.0)$ \\
Y-glutamyl transferase increased & $1(5.0)$ & $1(5.0)$ \\
Diarrhea & $1(5.0)$ & $1(5.0)$ \\
Autoimmune colitis & $1(5.0)$ & $1(5.0)$ \\
Pneumonitis & $1(5.0)$ & $1(5.0)$ \\
\hline Listed are any grade TRAE found in $\geq 10 \%$ patients, and all grade $3-4$ TRAEs
\end{tabular}

In the present study, after treatment with sintilimab plus CapeOx, patients with unresectable G/GEJ adenocarcinoma obtained an ORR of $85.0 \%$ (95\% CI: $62.1-$ 96.8\%), which is higher than that of conventional first line chemotherapy. For G/GEJ adenocarcinoma, firstline treatment mainly involves platinum-based chemotherapy and fluoropyrimidine [25]. The ORR of capecitabine-based or oxaliplatin-based therapies for G/GEJ adenocarcinoma was about 30-40\% [27, 28]. The ORR for anti-PD-1 antibodies with a chemotherapy regimen were variable. In the KEYNOTE-059 study, the ORR was $60.0 \%$ (95\% CI: $39.0-79.0 \%$ ) for pembrolizumab plus cisplatin/5-fluorouracilm (5-FU) as first-line treatment [29]. In the KEYNOTE-062 study, ORRs were 48.6 and $52.5 \%$ in patients with $\mathrm{a} \geq 1$ and $\geq 10$ combined positive score (CPS), respectively, after they received pembrolizumab plus cisplatin/5-FU or capecitabine regimen as first-line therapy [31]. In ATTRACTION-04, the ORR for nivolumab with S-1/ oxaliplatin was $57.1 \%$ (95\% CI: $34.0-78.2 \%$ ) and the ORR for nivolumab with CapeOx was 76.5\% (95\% CI: 50.1-93.2\%) [30]. In another study, the ORR was 


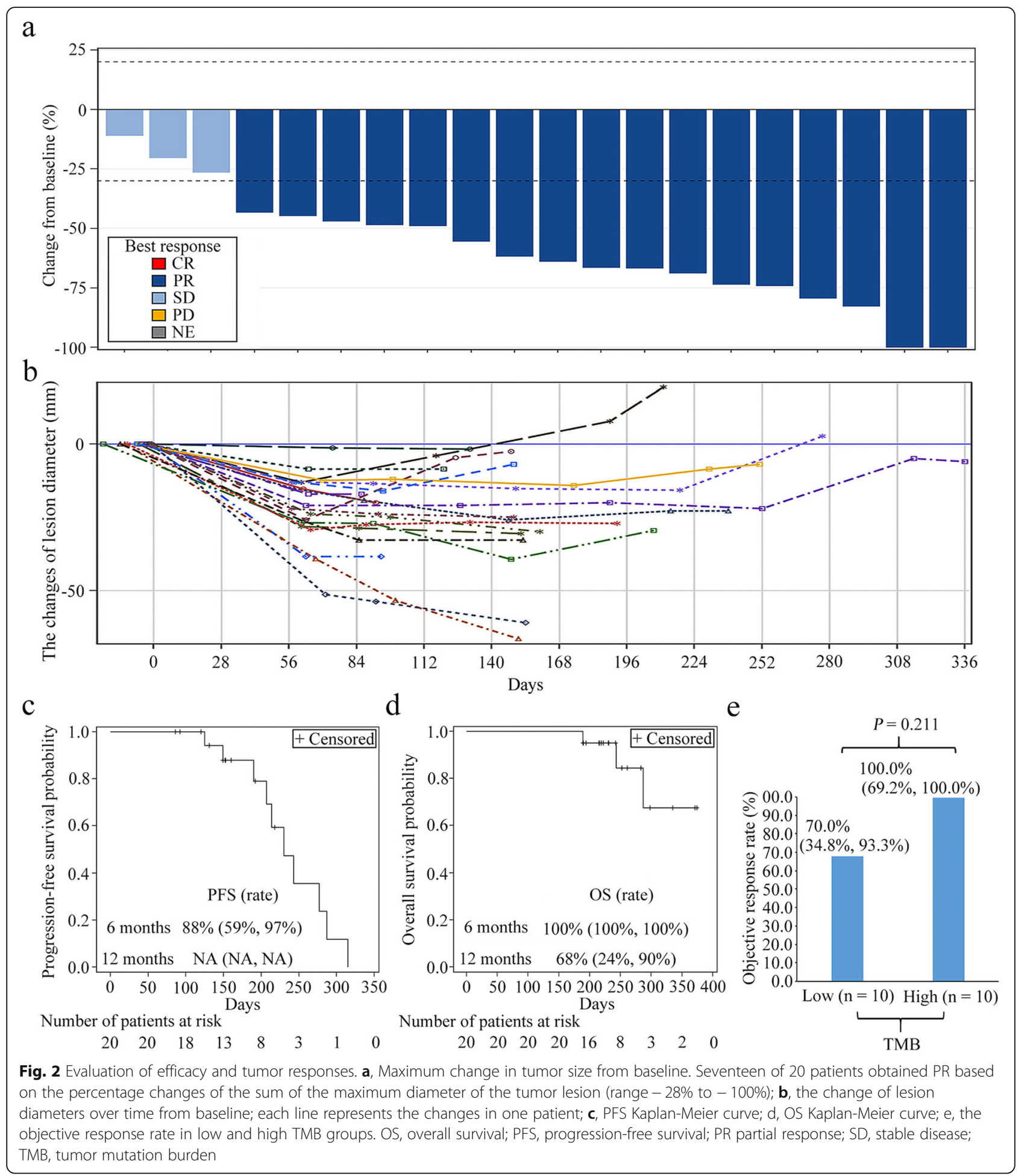

reported to be $66.7 \%$ for an anti-PD1 antibody toripalimab plus CapeOx treatment [32].

Sintilimab plus CapeOx also showed favorable longterm efficacy. Median PFS was 7.5 months (95\% CI: 6.29.4) and the 6-month PFS rate was $88 \%$. Median OS was not reached and the 6-month and 12- month OS rates were 100.0 and $68.0 \%$ respectively, which was higher than for conventional treatments with a median PFS of 5.6 months (95\% CI: 5.1-5.7) for capecitabine-cisplatin regimen [27] and a median OS of 11.3 months $(95 \%$ CI 9.6-13.0) for an epirubicin-oxaliplatin-capecitabine regimen [28]. The median PFS times for anti-PD-1 
Table 3 Efficacy evaluation of sintilimab

\begin{tabular}{lll}
\hline Efficacy evaluation & $\mathrm{n}$ & $\%$ \\
\hline $\mathrm{CR}$ & 0 & 0 \\
$\mathrm{PR}$ & 17 & $85.0 \%$ \\
$\mathrm{SD}$ & 3 & $15.0 \%$ \\
$\mathrm{PD}$ & 0 & 0 \\
ORR (95\% Cl) & 17 & $85.0 \%(95 \% \mathrm{Cl}:$ 62.1-96.8\%) \\
DCR (95\% Cl) & 20 & $100.0 \%(95 \% \mathrm{Cl}: 83.2-100.0 \%)$ \\
\hline
\end{tabular}

$C l$ confidence interval, $C R$ complete response, $D C R$ disease control rate, $O R R$ overall response rate, $P D$ progressive disease, $P R$ partial response, $S D$ stable disease

antibodies with a chemotherapy regimen were variable ranging from 5.7 to 10.6 months, a finding which might be associated with different populations and disease status [30-32].

Next-generation sequencing (NGS) has researcher enabled to perform target capture sequencing, which has been proposed as a reliable technique to identify mutated driver genes and for the estimation of TMBs. Its use has led to the detection of actionable alterations in various cancer related genes [33]. Regarding high TMB and the efficacy of PD-1 treatments, inconsistent results have been reported in previous studies. Wang et al. (2018) suggested that TMB might be associated with better efficacy for PD-1 monotherapy [32], whereas Mishima et al. [34] did not find a significant relationship between TMB and the response of gastric cancers to PD-1 therapy [34]. The latter data is in accordance with our finding that after treatment with sintilimab in combination with CapeOx, no significant difference in the clinical responses was found between $\mathrm{H}-\mathrm{TMB}$ and $\mathrm{L}$ $\mathrm{TMB}$ patients. However, using the median TMB as a cut-off is difficult to extrapolate to the real world clinic and bias due to the small sample size could not be excluded in the present study. In addition, it has been noted that up to now there is no uniform standard for H-TMB [33] and further investigations are urgently required.

\section{Conclusions}

Our results strongly indicate that sintilimab combined with CapeOx is an option for the first-line treatment of patients with advanced or metastatic G/GEJ adenocarcinoma. However, the sample size was small and it was a single-arm study without a comparator. The large scale, doubleblinded and randomized Phase III clinical trial ORIENT-16 for previously untreated advanced G/GEJ adenocarcinoma patients is being conducted to evaluate the efficacy and safety of sintilimab combined with CapeOx vs CapeOx alone (ClinicalTrials.gov Identifier: NCT03745170).

\section{Supplementary information}

Supplementary information accompanies this paper at https://doi.org/10. 1186/s12885-020-07251-z.

Additional file 1: Table S1. TNM stages of each patient. Table S2. Sintilimab related adverse events. Table S3. Chemotherapy-related adverse events. Appendix 1. Inclusion and exclusion criteria.

\section{Abbreviations \\ AEs: Adverse events; CapeOx: Oxaliplatin/capecitabine; CPS: Combined positive score; CR: Complete response; CT: Computed tomography; DCR: Disease control rate; DOR: Duration of response; ECOG-PS: Eastern Tumor Collaborative Group Performance Status; G: Gastric; GC: Gastric cancer; GEJ: Gastroesophageal junction; HER2: Human epidermal growth factor receptor-2; H-TMB: High tumor mutation burden; L-TMB: Low tumor mutation burden; Mb: Megabase; MRI: Magnetic resonance imaging; NGS: Next-generation sequencing; ORR: Objective response rate; OS: Overall survival; PD: Progressive disease; PD-1: Programmed death-1; PD-L1: Protein programmed death-ligand 1; PFS: Progression-free survival; PR: Partial response; SD: Stable disease; TMB: Tumor mutation burden; TNM: Tumor, nodes and metastases; TRAEs: Treatment-related AEs; TTR: Time to response; UICC: Union for International Cancer Control.}

\section{Acknowledgements}

We thank the patients and their families and the participating study teams for making this study possible and Yuan Fang (Innovent Biologics, Inc., Suzhou, China) for assistance with drafting the manuscript.

\section{Authors' contributions}

$H P J, N X, L S$ and $H Z$ were responsible for the design of the study. HPJ, YLZ, JQ, CM, XX, NL, CX, HW, LST and LS were responsible for acquisition of data; furthermore, NX, LS, HPJ, SYW and DLZ were in charge of analysis and interpretation of data. HPJ drafted the manuscript; NX, LS, HZ, SYW and DLZ revised and commented the draft. BP conducted the biomarker analysis. All authors read and approved the final manuscript.

\section{Funding}

The study was sponsored by Innovent Biologics, Inc., and co-funded by Eli Lilly and company. The study was also supported by the National Health and Family Planning Commission Research Fund \& Zhejiang Provincial Medical and Health major Science and Technology Plan Project (Grant No. KWJ-ZJ1802) and the Public Welfare Technology Application Research Project of Zhejiang Province (Grant No. LGF20E030004). Innovent Biologics, Inc. involved in the design of the study and collection, analysis, and interpretation of data and in writing the manuscript. All remaining funding bodies had no role in the design of the study and collection, analysis, and interpretation of data and in writing the manuscript.

\section{Availability of data and materials}

The datasets generated and/or analyzed during the current study are not publicly available since the new drug is being submitted to the National Medical Products Administration for approval, but are available from the corresponding author on reasonable request.

\section{Ethics approval and consent to participate}

The trial protocol was approved by the Institutional Review Boards and Ethical Committee of The First Hospital Affiliated to Zhejiang University School of Medicine and Beijing Cancer Hospital, and the study was carried out strictly following the declaration of Helsinki principles; all participating patients signed consent forms before taking part in the trial. The trial national registration number is NCT02937116.

\section{Consent for publication}

Not applicable.

\section{Competing interests}

Hui Zhou, Shuyan Wang, Donglei Zhu, Bo Peng are the staff of Innovent Biologics, Inc., Suzhou, China Suzhou, China. Lin Shen is the associate editor of BMC Cancer. All remaining authors declare that they have no competing interests. 


\section{Author details}

'Department of Medical Oncology, The First Affiliated Hospital, School of Medicine, Zhejiang University, No. 79, 86 Qingchun Road, Hangzhou 310003, China. ${ }^{2}$ Department of Surgical Oncology, The First Affiliated Hospital, School of Medicine, Zhejiang University, Hangzhou, China. ${ }^{3}$ Department of Medical Science and Strategy Oncology, Innovent Biologics, Inc, Suzhou, China. ${ }^{4}$ Department of Translational Medicine, Innovent Biologics, Inc, Suzhou, China. ${ }^{5}$ Department of Medical Oncology, Beijing Cancer Hospital, Beijing, China.

Received: 24 March 2020 Accepted: 3 August 2020

Published online: 14 August 2020

\section{References}

1. Bray F, Ferlay J, Soerjomataram I, Siegel RL, Torre LA, Jemal A. Global cancer statistics 2018: GLOBOCAN estimates of incidence and mortality worldwide for 36 cancers in 185 countries. CA Cancer J Clin. 2018;68(6):394-424.

2. Chen W, Zheng R, Baade PD, Zhang S, Zeng H, Bray F, et al. Cancer statistics in China, 2015. CA Cancer J Clin. 2016;66(2):115-32.

3. Bickenbach K, Strong VE. Comparisons of gastric Cancer treatments: east vs. West J Gastric Cancer. 2012;12(2):55-62.

4. Lui FH, Tuan B, Swenson SL, Wong RJ. Ethnic disparities in gastric cancer incidence and survival in the USA: an updated analysis of 1992-2009 SEER data. Dig Dis Sci. 2014;59(12):3027-34.

5. Ye XS, Yu C, Aggarwal A, Reinhard C. Genomic alterations and molecular subtypes of gastric cancers in Asians. Chin J Cancer. 2016:35(1):42.

6. Van Cutsem E, Moiseyenko VM, Tjulandin S, Majlis A, Constenla M, Boni C, et al. Phase III study of docetaxel and cisplatin plus fluorouracil compared with cisplatin and fluorouracil as first-line therapy for advanced gastric cancer: a report of the V325 study group. J Clin Oncol. 2006;24(31):4991-7.

7. Shah MA, Janjigian YY, Stoller R, Shibata S, Kemeny M, Krishnamurthi S, et al. Randomized multicenter phase II study of modified Docetaxel, Cisplatin, and fluorouracil (DCF) versus DCF plus growth factor support in patients with metastatic gastric adenocarcinoma: a study of the US gastric Cancer consortium. J Clin Oncol. 2015:33(33):3874-9.

8. Al-Batran SE, Hartmann JT, Probst S, Schmalenberg H, Hollerbach S, Hofheinz R, et al. Phase III trial in metastatic gastroesophageal adenocarcinoma with fluorouracil, leucovorin plus either oxaliplatin or cisplatin: a study of the Arbeitsgemeinschaft Internistische Onkologie. J Clin Oncol. 2008;26(9):1435-42.

9. Kang YK, Kang WK, Shin DB, Chen J, Xiong J, Wang J, et al. Capecitabine/ cisplatin versus 5 -fluorouracil/cisplatin as first-line therapy in patients with advanced gastric cancer: a randomised phase III noninferiority trial. Ann Oncol. 2009:20(4):666-73.

10. Bang YJ, Van Cutsem E, Feyereislova A, Chung HC, Shen L, Sawaki A, et al. Trastuzumab in combination with chemotherapy versus chemotherapy alone for treatment of HER2-positive advanced gastric or gastrooesophageal junction cancer (ToGA): a phase 3, open-label, randomised controlled trial. Lancet. 2010;376(9742):687-97.

11. Wei SC, Duffy CR, Allison JP. Fundamental mechanisms of immune checkpoint blockade therapy. Cancer Discov. 2018;8(9):1069-86.

12. Park YJ, Kuen DS, Chung Y. Future prospects of immune checkpoint blockade in cancer: from response prediction to overcoming resistance. Exp Mol Med. 2018;50(8):109.

13. Marin-Acevedo JA, Dholaria B, Soyano AE, Knutson KL, Chumsri S, Lou Y. Next generation of immune checkpoint therapy in cancer: new developments and challenges. J Hematol Oncol. 2018;11(1):39.

14. Lowther DE, Goods BA, Lucca LE, Lerner BA, Raddassi K, van Dijk D, et al. PD-1 marks dysfunctional regulatory $T$ cells in malignant gliomas. JCl Insight. 2016;1(5):e85935.

15. Dong H, Strome SE, Salomao DR, Tamura H, Hirano F, Flies DB, et al. Tumorassociated B7-H1 promotes T-cell apoptosis: a potential mechanism of immune evasion. Nat Med. 2002;8(8):793-800.

16. Muro K, Chung HC, Shankaran V, Geva R, Catenacci D, Gupta S, et al. Pembrolizumab for patients with PD-L1-positive advanced gastric cancer (KEYNOTE-012): a multicentre, open-label, phase 1b trial. Lancet Oncol. 2016; 17(6):717-26.

17. Fuchs CS, Doi T, Jang RW, Muro K, Satoh T, Machado M, et al. Safety and efficacy of Pembrolizumab Monotherapy in patients with previously treated advanced gastric and Gastroesophageal junction Cancer: phase 2 clinical KEYNOTE-059 trial. JAMA Oncol. 2018;4(5):e180013.
18. Kang YK, Boku N, Satoh T, Ryu MH, Chao Y, Kato K, et al. Nivolumab in patients with advanced gastric or gastro-oesophageal junction cancer refractory to, or intolerant of, at least two previous chemotherapy regimens (ONO-4538-12, ATTRACTION-2): a randomised, double-blind, placebocontrolled, phase 3 trial. Lancet. 2017;390(10111):2461-71.

19. Song M, Chen X, Wang L, Zhang Y. Future of anti-PD-1/PD-L1 applications: combinations with other therapeutic regimens. Chin J Cancer Res. 2018; 30(2):157.

20. Gandhi L, Rodriguez-Abreu D, Gadgeel S, Esteban E, Felip E, De Angelis F, et al. Pembrolizumab plus chemotherapy in metastatic non-small-cell lung Cancer. N Engl J Med. 2018:378(22):2078-92.

21. Paz-Ares L, Luft A, Vicente D, Tafreshi A, Gumus M, Mazieres J, et al. Pembrolizumab plus chemotherapy for squamous non-small-cell lung Cancer. N Engl J Med. 2018;379(21):2040-51.

22. Yan Y, Kumar AB, Finnes H, Markovic SN, Park S, Dronca RS, et al. Combining immune checkpoint inhibitors with conventional Cancer therapy. Front Immunol. 2018:9:1739.

23. Wang J, Fei K, Jing H, Wu Z, Wu W, Zhou S, et al. Durable blockade of PD-1 signaling links preclinical efficacy of sintilimab to its clinical benefit. MAbs. 2019;11(8):1443-51.

24. Brierley JD, Gospodarowicz MK, Wittekind C. TNM classification of malignant tumours. 8th ed. Oxford: Wiley; 2017.

25. National Comprehensive Cancer Network. Guidelines for Gastric Cancer Version 2: 2019. p. 2019

26. Cunningham D, Starling N, Rao S, Iveson T, Nicolson M, Coxon F, et al. Capecitabine and oxaliplatin for advanced esophagogastric cancer. N Engl J Med. 2008;358(1):36-46.

27. Lordick F, Kang YK, Chung HC, Salman P, Oh SC, Bodoky G, et al. Capecitabine and cisplatin with or without cetuximab for patients with previously untreated advanced gastric cancer (EXPAND): a randomised, open-label phase 3 trial. Lancet Oncol. 2013;14(6):490-9.

28. Waddell T, Chau I, Cunningham D, Gonzalez D, Okines AF, Okines C, et al. Epirubicin, oxaliplatin, and capecitabine with or without panitumumab for patients with previously untreated advanced oesophagogastric cancer (REAL3): a randomised, open-label phase 3 trial. Lancet Oncol. 2013;14(6):481-9.

29. Bang YJ, Kang YK, Catenacci DV, Muro K, Fuchs CS, Geva R, et al. Pembrolizumab alone or in combination with chemotherapy as first-line therapy for patients with advanced gastric or gastroesophageal junction adenocarcinoma: results from the phase II nonrandomized KEYNOTE-059 study. Gastric Cancer. 2019;22(4):828-37.

30. Boku N, Ryu MH, Kato K, Chung HC, Minashi K, Lee KW, et al. Safety and efficacy of nivolumab in combination with $\mathrm{S}-1$ /capecitabine plus oxaliplatin in patients with previously untreated, unresectable, advanced, or recurrent gastric/gastroesophageal junction cancer: interim results of a randomized, phase II trial (ATTRACTION-4). Ann Oncol. 2019;30(2):250-8.

31. Tabernero J, Cutsem EV, Bang YJ, Fuchs CS, Wyrwicz L, Lee K-W, et al. Pembrolizumab with or without chemotherapy versus chemotherapy in advanced G/GEJ adenocarcinoma the phase 3, keynote-062 study. J Clin Oncol. 2019;37(18):suppl.LBA4007.

32. Wang F, Wei XL, Wang FH, Xu N, Shen L, Dai GH, et al. Safety, efficacy and tumor mutational burden as a biomarker of overall survival benefit in chemo-refractory gastric cancer treated with toripalimab, a PD1 antibody in phase Ib/II clinical trial NCT02915432. Ann Oncol. 2019;30(9):1479-86.

33. Cai H, Jing C, Chang X, Ding D, Han T, Yang J, et al. Mutational landscape of gastric cancer and clinical application of genomic profiling based on target next-generation sequencing. J Transl Med. 2019;17(1):189.

34. Mishima S, Kawazoe A, Nakamura Y, Sasaki A, Kotani D, Kuboki Y, et al. Clinicopathological and molecular features of responders to nivolumab for patients with advanced gastric cancer. J Immunother Cancer. 2019;7(1):24.

\section{Publisher's Note}

Springer Nature remains neutral with regard to jurisdictional claims in published maps and institutional affiliations. 